\title{
Prophylactic Erythropoietin for Neuroprotection in Very Preterm Infants: A Meta-Analysis Update
}

\author{
Hendrik S. Fischer, Nora J. Reibel, Christoph Bührer and Christof Dame* \\ Department of Neonatology, Charité-Universitätsmedizin Berlin, Berlin, Germany
}

A meta-analysis update of randomized controlled trials investigating recombinant human erythropoietin suggests improved neurodevelopmental outcome in preterm infants. There was substantial heterogeneity, which could be ascribed to a single trial. Exclusion of this trial featuring a high risk of bias abolished heterogeneity and any effects of recombinant human erythropoietin treatment.

Keywords: erythropoietin, meta-analysis, neurodevelopment, neuroprotection, VLBW and ELBW infants

\section{INTRODUCTION}

\section{OPEN ACCESS}

Edited by:

Graeme R. Polglase,

Monash University, Australia

Reviewed by:

Helen G. Liley,

The University of Qeensland, Australia

Robin Ohls,

The University of Utah, United States

Hans Ulrich Bucher,

University Hospital Zürich, Switzerland

*Correspondence:

Christof Dame

christof.dame@charite.de

Specialty section:

This article was submitted to Neonatology,

a section of the journal

Frontiers in Pediatrics

Received: 22 January 2021 Accepted: 31 March 2021 Published: 20 May 2021

Citation:

Fischer HS, Reibel NJ, Bührer C and Dame C (2021) Prophylactic Erythropoietin for Neuroprotection in Very Preterm Infants: A Meta-Analysis Update. Front. Pediatr. 9:657228. doi: 10.3389/fped.2021.657228
Recombinant human erythropoietin (rhEPO) is considered a neuroprotective or neurorestorative drug for the premature brain, because it has been experimentally shown to prevent or to mitigate white matter injury. rhEPO reduces the need for red blood cell transfusions in preterm infants, but only few randomized controlled trials (RCTs) have addressed neurodevelopmental outcomes. Our previous meta-analysis (2017) showed a beneficial effect of prophylactic rhEPO on cognitive scores at 18-24 months' corrected age (1). The recent publication of a large multicenter RCT, Preterm Erythropoietin Neuroprotection Trial (PENUT), raises the question as to the robustness of this finding (2).

\section{METHODS}

Following the original methodology (1), we aimed to identify all RCTs investigating the effects of prophylactic rhEPO in preterm infants vs. no treatment or placebo and reporting neurodevelopmental outcomes. The literature search, study selection, and data extraction followed the standard search methods of the Cochrane Collaboration (3). The database search was updated on October 11, 2020 (see Supplementary Information A for details). Two authors (H.F. and C.D.) independently searched the databases MEDLINE, Embase, and CENTRAL and used additional information (cross-referencing of previous reviews and trials, expert information, information about ongoing trials of the International Clinical Trials Registry Platform (4) to identify and select studies and extracted the data). The included studies were independently assessed by two study authors (H.F. and N.R.) using the Cochrane risk-of-bias tool for RCTs. Any discrepancies were resolved by a third author (C.B. or C.D.). For all pre-specified outcomes, funnel plots were used to assess for publication bias (3).

The primary outcome was the number of infants with a Mental Development Index (MDI) $<70$ on the Bayley Scales of Infant Development, second edition (BSID-II), or a composite cognitive score $<85$ on BSID-III. Equivalence of these cognitive outcome measures was accepted, as a study showed that a composite cognitive score $<85$ (BSID-III) predicted an MDI $<70$ (BSID-II) with an overall agreement of $97.3 \%$ (5). Secondary outcomes included the number of infants with a Psychomotor Development Index $<70$ (BSID-II), cerebral palsy, any neurodevelopmental impairment, and a planned subgroup analysis of 
TABLE 1 | Characteristics of included studies.

\begin{tabular}{|c|c|c|c|c|c|c|}
\hline References & Year & $n$ & $\begin{array}{l}\text { Gestational age, } \\
\text { birth weight }\end{array}$ & $\begin{array}{l}\text { Time point of } \\
\text { intervention }\end{array}$ & Intervention & Recruitment \\
\hline Ohls et al. (7) & 2004 & 102 & $\leq 32 \% / 7, \leq 1,000 \mathrm{~g}$ & 24-96h of age & $\begin{array}{l}\text { rhEPO } 400 \mathrm{IU} / \mathrm{kg} \text { IV or SC, } 3 \text { times per week } \\
\text { until } 35 \text { \% /7 weeks' postmenstrual age }\end{array}$ & 1997-1998 \\
\hline Ohls et al. (8) & 2014 & $53^{a}$ & any $\mathrm{GA},{ }^{\mathrm{b}} 500-1,250 \mathrm{~g}$ & $\leq 48 \mathrm{~h}$ of age & $\begin{array}{l}\text { rhEPO } 400 \text { IU/kg SC, } 3 \text { times per week until } 35 \\
\text { \%/7 weeks' postmenstrual age }\end{array}$ & 2006-2010 \\
\hline Natalucci et al. (9) & 2016 & 365 & $\begin{array}{l}26 \% \text { to } 31^{6 / 7} \text {, any } \\
\text { BW }\end{array}$ & $<3 h$ of age & $\begin{array}{l}\mathrm{rhEPO} 3,000 \mathrm{IU} / \mathrm{kg} \text { IV at }<3,12-18 \text {, and } \\
36-42 \mathrm{~h} \text { of age }\end{array}$ & 2005-2012 \\
\hline Song et al. (10) & 2016 & 613 & $\leq 32 \%$, any BW & $<72 \mathrm{~h}$ of age & rhEPO 500 IU/kg IV every other day for 2 weeks & 2009-2013 \\
\hline Peltoniemi et al. (6) & 2017 & 35 & $\begin{array}{l}24 \% \text { to } 30 \% / 7 \\
700-1,500 \mathrm{~g}\end{array}$ & 1st day of life & rhEPO 250 IU/kg IV daily from days 1 to 6 & 1998-2000 \\
\hline Juul et al. (2) & 2020 & 628 & $\begin{array}{l}24 \% \text { to } 27^{6 / 7} \text {, any } \\
\text { BW }\end{array}$ & $\leq 24 \mathrm{~h}$ of age & $\begin{array}{l}\text { rhEPO } 1,000 \text { IU/kg IV every } 48 \text { h for } 6 \text { doses, } \\
\text { followed by } 400 \text { IU SC } 3 \text { times per week until } \\
32 \text { 6/7 weeks' postmenstrual age }\end{array}$ & 2013-2016 \\
\hline
\end{tabular}

BW, birth weight; GA, gestational age; rhEPO, recombinant human erythropoietin; IU, international units; IV, intravenously; SC, subcutaneously.

a This study had three groups: rhEPO $(n=29)$ vs. placebo $(n=24)$ vs. darbepoetin $(n=27)$. The darbepoetin group was not included in the meta-analysis.

${ }^{b}$ Median (interquartile range) GA of included infants: 28 (26-29) weeks; study entry criteria: preterm infants with a birth weight of 500-1,250 g.

MDI $<70$ (BSID-II) or composite cognitive score $<85$ (BSID-III) in infants of $<28$ weeks' gestational age.

To include PENUT, the timeframe was extended to $18-26$ months' corrected age (previously 18-24 months). Moreover, we added (1) a sensitivity analysis that excluded the data from RCTs with a high risk of bias in one or more domains of the Cochrane risk-of-bias assessment tool; (2) exploratory analyses that investigated the effects of prophylactic rhEPO on mortality, on a combined outcome of death or MDI $<70$ or composite cognitive score $<85$ and on a combined outcome of death or any neurodevelopmental impairment; and (3) exploratory analyses that investigated the effect of high-dose $\mathrm{rhEPO}(\geq 1,000 \mathrm{iU} / \mathrm{kg}$ per dose) vs. low- to moderate-dose rhEPO $(<1,000 \mathrm{iU} / \mathrm{kg}$ per dose) on neurodevelopmental outcome measures. A level of statistical significance of $p<0.05$ was accepted when testing for subgroup differences.

\section{RESULTS}

The update identified 164 additional records, of which 11 were assessed as full text (Supplementary Figure 1): Six RCTs were excluded because they did not report neurodevelopmental outcomes, and three reported behavioral measures or results of imaging studies at 3.5-6 years (see Supplementary Information B for details). Two more RCTs $(2,6)$ were included (Table 1). Study details and the complete risk-of-bias assessment are available online (Supplementary Table 1).

Meta-analysis of the five trials that reported MDI $<70$ (BSID II) or composite cognitive score $<85$ (BSID-III) at 18-26 months' corrected age showed a risk reduction from 20 to $14 \%$ associated

Abbreviations: BSD-II, Bayley Scales of Infant Development, second edition; BSD-III, Bayley Scales of Infant Development, third edition; MDI, Mental Development Index; PENUT, Preterm Erythropoietin Neuroprotection Trial; $\mathrm{RBC}$, red blood cell; RCT, randomized controlled trial; rhEPO, recombinant human erythropoietin. with prophylactic rhEPO (odds ratio $0.61, p=0.03$, Figure 1A), corresponding to a number needed to treat of 17. rhEPO had no significant effect on any secondary outcome. Forest plots for outcomes with new data are shown in Figures 1B-D.

The methodological quality of the included RCTs was mostly high, with low or sometimes unclear risk of bias in most domains (Supplementary Table 1). Only one study (10) was categorized as "high risk of bias" in two domains (blinding of participants and personnel and selective reporting). After exclusion of this study, the statistically significant effect of rhEPO on the primary outcome of MDI $<70$ or a composite cognitive score $<85$ disappeared (Supplementary Figure 2), as did all indicators of heterogeneity in this analysis $\left(\tau^{2}=0, \chi^{2}<d f, I^{2}=0 \%\right)$.

Exploratory analyses showed no effect of rhEPO on mortality, on the combined outcome of death or MDI $<70$ or a composite cognitive score $<85$ or on the combined outcome of death or neurodevelopmental impairment (Supplementary Figure 3). The exploratory subgroup analyses showed a beneficial effect of rhEPO on MDI $<70$ or a composite cognitive score $<85$ and a borderline beneficial effect on any neurodevelopmental impairment, only in the subgroup of trials that applied lowto moderate-dose rhEPO (Supplementary Figures 4A,D). The tests for subgroup differences showed no differences in the effects of high-dose vs. low- to moderate-dose rhEPO on any neurodevelopmental outcome (Supplementary Figure 4). The assessment of the funnel plots did not reveal major asymmetries (Supplementary Figure 5).

\section{DISCUSSION}

This update continues to indicate a benefit of prophylactic rhEPO by lowering the number of very preterm infants with an MDI of $<70$ or composite cognitive score of $<85$ (Figure 1A). However, no effect on MDI $<70$ or a composite cognitive score $<85$ could be ascertained in the subgroup of infants born below 28 weeks' gestational age, possibly due to the small number of study 
A $\quad$ MDI $<70$ (BSID II) or composite cognitive score $<85$ (BSID III)

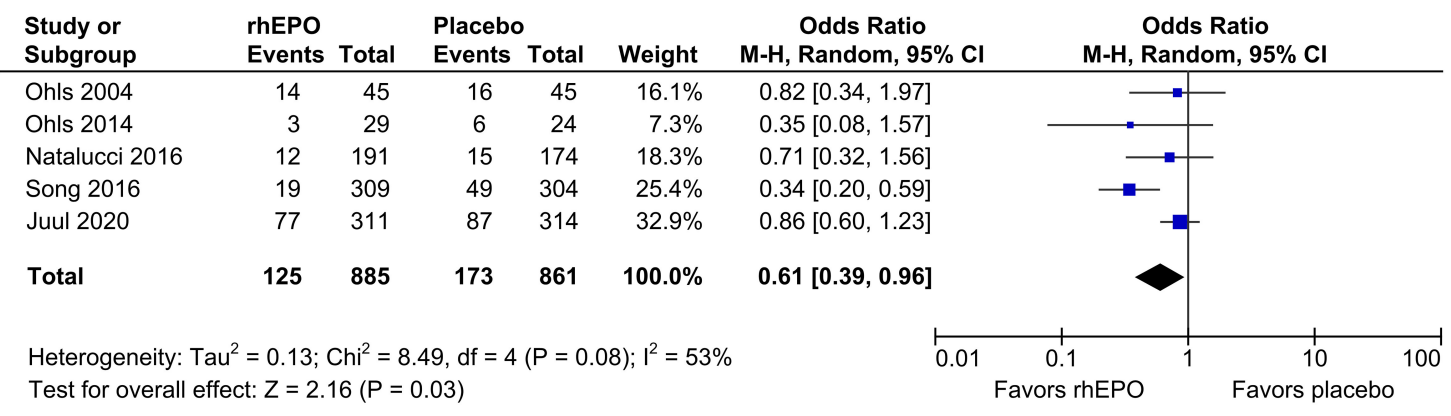

B $\mathrm{MDI}<70$ (BSID II) or composite cognitive score <85 (BSID III) in infants <28 weeks' gestational age

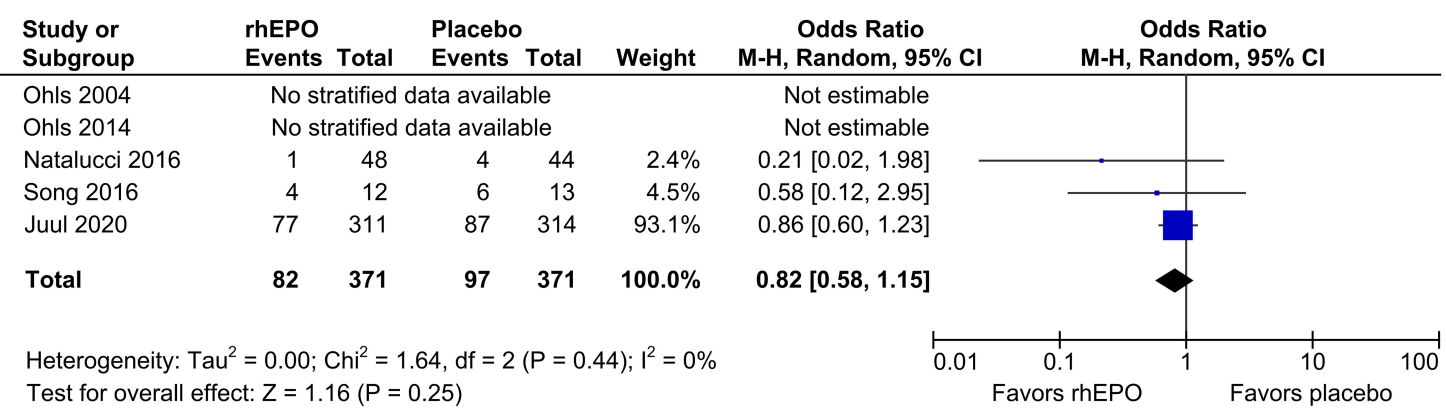

C Cerebral palsy

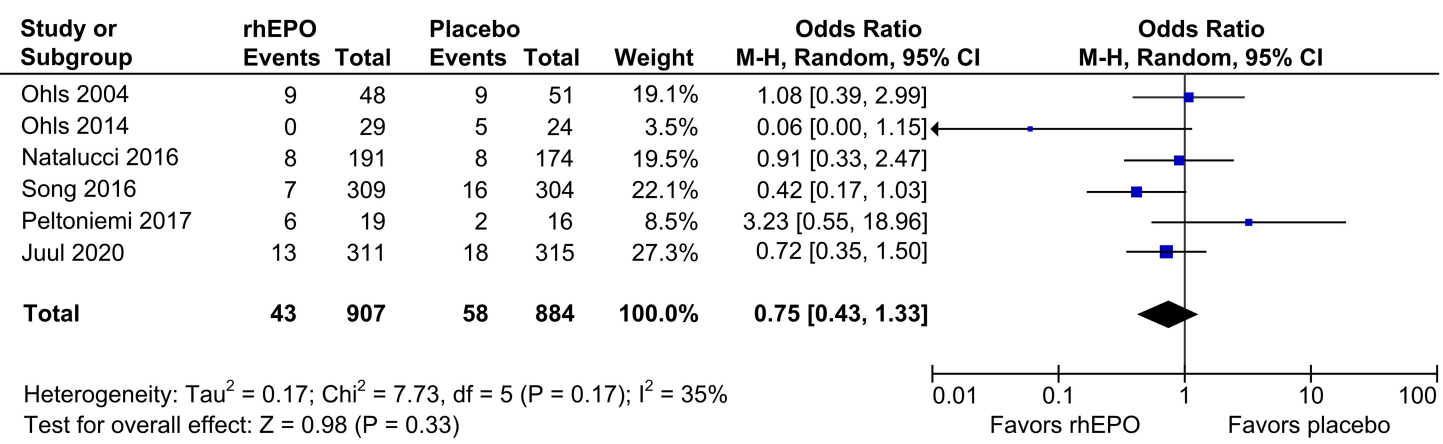

D Any neurodevelopmental impairment

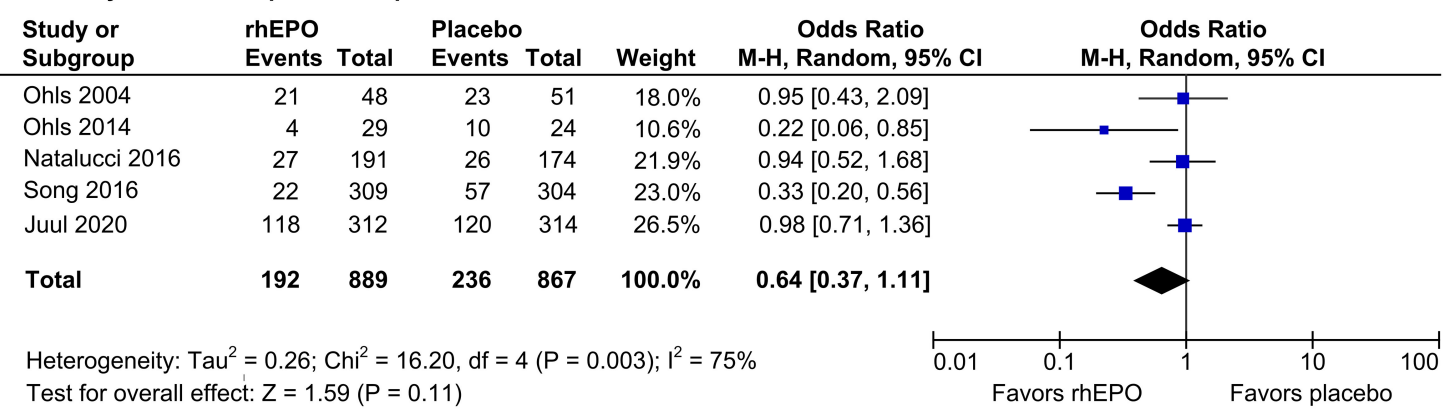

FIGURE 1 | Effects of rhEPO on neurodevelopment at 18-26 months' corrected age. Forest plots show the effects on the number of infants with an MDI <70 (BSID-II) or a composite cognitive score $<85$ (BSID-III) in all infants (primary outcome, (A) and in infants $<281 / 7$ weeks' gestational age (planned subgroup analysis, (B), on cerebral palsy (C), and on any neurodevelopmental impairment (D). M-H, Mantel-Haenszel. 
patients in this subgroup (Figure 1B). There were no beneficial effects on any secondary outcomes. While significantly improved neurodevelopment at the age of 3.5 to 4 years has been reported in one RCT (11), prophylactic high-dose rhEPO had no effect on cognitive scores at 5 years of age in a large Swiss RCT (12).

The results of the present meta-analysis may have been influenced by publication bias, because most rhEPO RCTs identified in the literature search did not report neurodevelopmental follow-up data. In this setting, the funnel plots were not conspicuous but had a low power to detect publication bias, because a maximum number of 6 RCTs were included in the meta-analysis update (Supplementary Figure 5). Moreover, the uncertainty as to the effects of rhEPO is fostered by the moderate to substantial heterogeneity of the RCTs included in our meta-analysis $\left(I^{2}=53 \%\right.$, Figure 1A; $I^{2}=$ $75 \%$, Figure 1D). Statistical heterogeneity and the presumed effect of rhEPO on MDI $<70$ or a composite cognitive score $<85$ disappeared after exclusion of a single study (10) featuring increased risk of bias (Supplementary Figure 2A). Discrepancies with the main analysis (Figure 1A) may not necessarily result from bias in the excluded RCT but could reside in differences in the study protocol, in the healthcare environment, or in the population studied.

Looking at the differences between the study protocols, it becomes obvious that quite different dosing regimens of rhEPO were applied (Table 1), depending upon the concept of using rhEPO with the primary intention of neuroprotection/neurorepair vs. prevention of anemia of prematurity or even a combined approach. Admittedly, a subgroup analysis on dosing effects remains very limited due to the heterogeneity of the study protocols. In an exploratory analysis, we compared neurodevelopmental outcomes in RCTs with early high-dose $\operatorname{rhEPO}(\geq 1,000 \mathrm{iU} / \mathrm{kg}$ per dose) intended for neuroprotection/neurorepair in accordance with experimental data on the transport of rhEPO across the blood-brain barrier (13) vs. low- to moderate-dose rhEPO $(<1,000 \mathrm{iU} / \mathrm{kg}$ per dose) intended for the prevention of red blood cell (RBC) transfusions. Although the beneficial effect of rhEPO on MDI $<70$ or a composite cognitive score $<85$ was preserved in the subgroup meta-analysis of trials that applied low- to moderate-dose rhEPO, the tests for subgroup differences indicated no statistically significant subgroup effect for any outcome (Supplementary Figure 4). Alternatively, the power of this analysis to detect subgroup differences may have

\section{REFERENCES}

1. Fischer HS, Reibel NJ, Bührer C, Dame C. Prophylactic early erythropoietin for neuroprotection in preterm infants: a meta-analysis. Pediatrics. (2017) 139:e20164317. doi: 10.1542/peds.2016-4317

2. Juul SE, Comstock BA, Wadhawan R, Mayock DE, Courtney SE, Robinson $\mathrm{T}$, et al. A randomized trial of erythropoietin for neuroprotection in preterm infants. N Engl J Med. (2020) 382:233-43. doi: 10.1056/NEJMoa190 7423

3. Higgins JPT, Green S (eds.). Cochrane Handbook for Systematic Reviews of Interventions. Version 5.1.0. The Cochrane Collaboration. (updated March 2011). been too small, because few trials were included and because less trials contributed data to the high-dose subgroup than to the low-dose subgroup (uneven covariate distribution). Considering the aforementioned uncertainties and the clinical context, we believe that the individual effect of RBC transfusion on neurodevelopment also deserves attention. Interestingly, the post hoc analysis of the PENUT trial indicated that each transfusion of RBCs was associated with a decrease in mean cognitive score, motor score, and language score (BSID-III). Moreover, significant negative associations between BSID-III scores, transfusion volume, and donor exposure were observed in the placebo group, but not in the rhEPO group (14). Since an early start of rhEPO therapy significantly reduced the number of RBC transfusions, as shown in the updated Cochrane Review and in the recent PENUT trial $(15,16)$, rhEPO may still reclaim its value in the patient blood management of very preterm infants.

Finally, a retrospective case-control study suggested that beneficial neurodevelopmental effects of rhEPO may not become apparent prior to preschool age and may be restricted to children who had suffered from intraventricular hemorrhage (17). Thus, we have to await the 5-years neurodevelopmental follow-up data (EpoRepair, NCT02076373) of infants with intraventricular hemorrhage randomized to rhEPO or placebo (18) to decide whether or not rhEPO may have a role in alleviating neurodevelopmental impairment due to a certain pathophysiology of white matter injury.

\section{AUTHOR CONTRIBUTIONS}

HF designed the meta-analysis, searched for relevant studies, extracted, assessed, and analyzed the data, and drafted the manuscript. NR and $\mathrm{CB}$ contributed to data extraction and assessment and critically revised the manuscript. CD conceptualized the meta-analysis, searched for relevant studies, extracted and assessed the data, and drafted the manuscript. All authors critically reviewed and revised the manuscript, approved the final version as submitted, and agreed to be accountable for all aspects of the work.

\section{SUPPLEMENTARY MATERIAL}

The Supplementary Material for this article can be found online at: https://www.frontiersin.org/articles/10.3389/fped. 2021.657228/full\#supplementary-material
4. World Health Organization. International Clinical Trial Registry Platform (ICTRP). Available online at: https://www.who.int/clinical-trials-registryplatform (accessed October 11, 2020).

5. Johnson S, Moore T, Marlow N. Using the bayley-iii to assess neurodevelopmental delay: which cut-off should be used? Pediatr Res. (2014) 75:670-4. doi: 10.1038/pr.2014.10

6. Peltoniemi OM, Anttila E, Kaukola T, Buonocore G, Hallman M. Randomized trial of early erythropoietin supplementation after preterm birth: iron metabolism and outcome. Early Hum Dev. (2017) 109:44-9. doi: 10.1016/j.earlhumdev.2017.04.001

7. Ohls RK, Ehrenkranz RA, Das A, Dusick AM, Yolton K, Romano E, et al. Neurodevelopmental outcome and growth at 18 to 22 months' corrected age 
in extremely low birth weight infants treated with early erythropoietin and iron. Pediatrics. (2004) 114:1287-91. doi: 10.1542/peds.2003-1129-L

8. Ohls RK, Kamath-Rayne BD, Christensen RD, Wiedmeier SE, Rosenberg A, Fuller J, et al. Cognitive outcomes of preterm infants randomized to darbepoetin, erythropoietin, or placebo. Pediatrics. (2014) 133:1023-30. doi: 10.1542/peds.2013-4307

9. Natalucci G, Latal B, Koller B, Rüegger C, Sick B, Held L, et al. Effect of early prophylactic high-dose recombinant human erythropoietin in very preterm infants on neurodevelopmental outcome at 2 years: A randomized clinical trial. JAMA. (2016) 315:2079-85. doi: 10.1001/jama.2016.5504

10. Song J, Sun H, Xu F, Kang W, Gao L, Guo J, et al. Recombinant human erythropoietin improves neurological outcomes in very preterm infants. Ann Neurol. (2016) 80:24-34. doi: 10.1002/ana.24677

11. Ohls RK, Cannon DC, Phillips J, Caprihan A, Patel S, Winter S, et al. Preschool assessment of preterm infants treated with darbepoetin and erythropoietin. Pediatrics. (2016) 137:e20153859. doi: 10.1542/peds.2015-3859

12. Natalucci G, Latal B, Koller B, Rüegger C, Sick B, Held L, et al. Neurodevelopmental outcomes at age 5 years after prophylactic early highdose recombinant human erythropoietin for neuroprotection in very preterm infants. JAMA. (2020) 324:2324-7. doi: 10.1001/jama.2020.19395

13. Juul SE, McPherson RJ, Farrell FX, Jolliffe L, Ness DJ, Gleason CA. Erytropoietin concentrations in cerebrospinal fluid of nonhuman primates and fetal sheep following high-dose recombinant erythropoietin. Biol Neonate. (2004) 85:138-44. doi: 10.1159/000074970

14. Vu PT, Ohls RK, Mayock DE, German KR, Comstock BA, Heagerty PJ, et al. Transfusions and neurodevelopmental outcomes in extremely low gestation neonates enrolled in the PENUT trial: a randomized clinical trial. Pediatr Res. (2021) 11:1-8. doi: 10.1038/s41390-020-01273-w
15. Juul SE, Vu PT, Comstock BA, Wadhawan R, Mayock DE, Courtney SE, et al. Effect of high-dose erythropoietin on blood transfusions in extremely low gestational age neonates: post hoc analysis of a randomized clinical trial. JAMA Pediatr. (2020) 174:933-43. doi: 10.1001/jamapediatrics.20 20.2271

16. Ohlsson A, Aher SM. Early erythropoiesis-stimulating agents in preterm or low birth weight infants. Cochrane Database Syst Rev. (2020) 2: CD004863. doi: 10.1002/14651858.CD0048 63.pub6

17. Neubauer AP, Voss W, Wachtendorf M, Jungmann T. Erythropoietin improves neurodevelopmental outcome of extremely preterm infants. Ann Neurol. (2010) 67:657-66. doi: 10.1002/ana.21977

18. Rüegger CM, Hagmann CF, Bührer C, Held L, Bucher HU, Wellmann S, et al. Erythropoietin for the repair of cerebral injury in very preterm infants (EpoRepair). Neonatology. (2015) 108:198-204. doi: 10.1159/0004 37248

Conflict of Interest: The authors declare that the research was conducted in the absence of any commercial or financial relationships that could be construed as a potential conflict of interest.

Copyright $\odot 2021$ Fischer, Reibel, Bührer and Dame. This is an open-access article distributed under the terms of the Creative Commons Attribution License (CC BY). The use, distribution or reproduction in other forums is permitted, provided the original author(s) and the copyright owner(s) are credited and that the original publication in this journal is cited, in accordance with accepted academic practice. No use, distribution or reproduction is permitted which does not comply with these terms. 